\title{
Dynamics of an acoustically trapped sphere in beating sound waves
}

\author{
Mohammed A. Abdelaziz $\bullet$ and David G. Grier $\odot$ \\ Department of Physics and Center for Soft Matter Research, New York University, New York, New York 10003, USA
}

(Received 29 August 2020; accepted 7 December 2020; published 26 January 2021; corrected 21 October 2021)

\begin{abstract}
A focused acoustic standing wave creates a Hookean potential well for a small sphere and can levitate it stably against gravity. Exposing the trapped sphere to a second transverse traveling sound wave imposes an additional acoustic force that drives the sphere away from its mechanical equilibrium. The driving force is shaped by interference between the standing trapping wave and the traveling driving wave. If, furthermore, the traveling wave is detuned from the standing wave, the driving force oscillates at the difference frequency. Far from behaving like a textbook-driven harmonic oscillator, however, the wave-driven harmonic oscillator instead exhibits a remarkably rich variety of dynamical behaviors. These include oscillations at both harmonics and subharmonics of the driving frequency, period-doubling routes to chaos, and Fibonacci cascades. This model system therefore illustrates opportunities for dynamic acoustic manipulation based on spectral, rather than spatial, control of the sound field.
\end{abstract}

DOI: 10.1103/PhysRevResearch.3.013079

\section{INTRODUCTION}

Acoustic manipulation is emerging as an attractive alternative to optical manipulation for applications where large forces are required to move sizable objects over macroscopic distances [1-5]. Sound waves can exert far more force per watt than light waves [6,7], but are not yet so easy to control. Optical techniques have the advantage in this regard thanks to a wealth of technology for controlling the spatial structure of laser beams. Dynamic holographic optical trapping, for example, uses megapixel arrays of phase-shifting elements to sculpt an ordinary laser beam into complex three-dimensional optical force fields [8,9]. Analogous devices for sound are under development $[2,10-12]$, but do not yet offer the same degree of control afforded by their optical counterparts.

One respect in which sound has a clear advantage over light is the ease with which a coherent sound wave's frequency can be altered [13]. The present study illustrates the opportunities for dexterous and dynamic acoustic manipulation afforded by spectral engineering through experimental and numerical studies of a deceptively simple dynamical system assembled and actuated by two continuous sound waves. Slightly detuning the two waves gives rise to a remarkably rich and potentially useful spatiotemporal phenomenology without requiring active control of the sound field's structure.

\section{OSCILLATIONS IN A BEATING ACOUSTIC TRAP}

Our system, shown schematically in Fig. 1(a), consists of a small sphere of mass $m$ that is stably levitated in air by

Published by the American Physical Society under the terms of the Creative Commons Attribution 4.0 International license. Further distribution of this work must maintain attribution to the author(s) and the published article's title, journal citation, and DOI. a vertical standing-wave acoustic trap at frequency $\omega$. The standing wave's transverse intensity gradients give rise to a restoring force that localizes the sphere in a three-dimensional harmonic well with natural frequency $\omega_{0} \ll \omega$. The trapped sphere also is exposed to a traveling sound wave at frequency $\omega+\Delta \omega$ that is projected horizontally. Interference between the two sound waves creates a force field for the sphere that oscillates at the difference frequency, $\Delta \omega$. Using the difference frequency between beating waves to achieve dynamical forcing appears not to have been considered previously [13]. Unlike conventional harmonic driving, the beat-field force depends on the driven particle's instantaneous position because the interference pattern moves through the system like a traveling wave. The state dependence of wave-mediated driving gives rise to a remarkably rich phenomenology including robust scanning modes that hold the promise of practical applications [11,12].

The vertical acoustic trap in our instrument is created by a piezoelectric transducer [Sunnytec Electronics; STC-4SH3540(B)] that is driven near its specified resonance frequency of $40 \pm 1 \mathrm{kHz}$ by a function generator (Stanford Research Systems; DS345). The transducer has a conical aluminum horn with a $4.5-\mathrm{cm}$-diameter output coupler that slightly concentrates the sound wave on the acoustic axis. This wave is reflected by a horizontal Plexiglas sheet to form a standingwave acoustic trap. The Plexiglas reflector is positioned around $6 \mathrm{~cm}$ above the transducer using a micrometer drive (Prior Scientific; FB201 Manual Focus Block). The reflector's position is adjusted to maximize the amplitude of the pressure wave at the reflector's surface, as measured by a piezoelectric transducer and a lock-in amplifier (Stanford Research Systems; SR830) referenced to the function generator.

The transverse traveling wave is created by a smaller piezoelectric transducer (American Piezo; 10-3155) with a specified resonance frequency of $40 \pm 1 \mathrm{kHz}$. This transducer is driven by a second function generator (Feeltech; FY6600) 


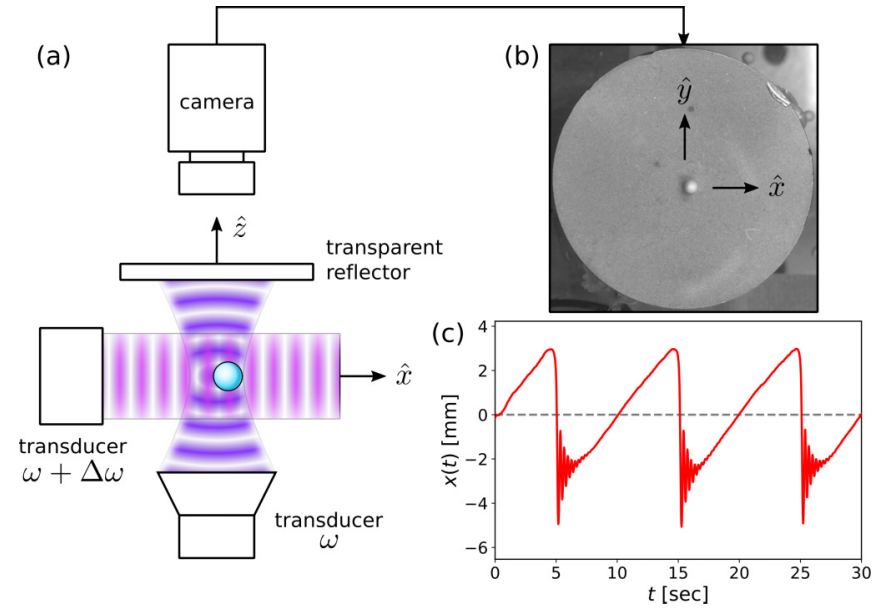

FIG. 1. (a) Schematic representation of an acoustically levitated particle driven by a harmonic traveling wave. (b) Video frame showing a top view of the particle at $z=4 \mathrm{~mm}$ above the transducer in a standing wave at $f=40.3 \mathrm{kHz}$. (c) Measured trajectory, $x(t)$, of the particle driven by a difference frequency, $\Delta f=0.1 \mathrm{~Hz}$.

whose time base is tied to the first to ensure relative phase stability. The frequency and amplitude of this wave are adjusted to control the dynamical state of the levitated bead.

The measured acoustic output of the trapping transducer is $63.5 \mathrm{PaV}^{-1} \mathrm{~m}^{-1}$ and trapping is performed with a driving signal of $5 \mathrm{~V}_{\mathrm{pp}}$. The orthogonal transducer provides an acoustic output of $1.38 \mathrm{PaV}^{-1} \mathrm{~m}^{-1}$ and is driven at $10 \mathrm{~V}_{\mathrm{pp}}$ to $40 \mathrm{~V}_{\mathrm{pp}}$. Both values for the measured acoustic output are consistent with the manufacturers' specifications. The pressure wave's amplitude is low enough that nonlinear effects may be ignored in the waves' interference $[14,15]$.

The transparent reflector also provides optical access, allowing us to record the sphere's motions in the horizontal plane at 40 frames/s with a magnification of $0.1 \mathrm{~mm} / \mathrm{pixel}$ using a vertically oriented video camera (FLiR; Flea 3 monochrome). Figure 1(b) presents one video frame [16] showing a 2-mm-diameter styrofoam bead stably levitated roughly $1 \mathrm{~mm}$ above the transducer's surface by a standing wave at the measured resonance frequency, $f=\omega /(2 \pi)=$ $40.3 \mathrm{kHz}$. The sphere reproducibly jumps into this node when the acoustic trap is energized, ensuring consistent trapping conditions from run to run.

The trace in Fig. 1(c) shows the bead's measured trajectory along $\hat{x}$ when the driving wave is detuned from the trapping wave by $\Delta f=\Delta \omega /(2 \pi)=0.1 \mathrm{~Hz}$. The bead's position in each video frame is monitored with $10 \mu \mathrm{m}$ precision using the TRACKPY particle-tracking library $[17,18]$. This trace embodies both the promise and the challenge of the spectral control afforded by acoustic forces. Rather than undergoing simple harmonic motion, the floating bead sweeps out a nearly linear sawtooth pattern with distinct ringing on the flyback. This mode of motion resembles the linear scanners that have been demonstrated with acoustic and optical holographic trapping $[11,19,20]$. Whereas holographic control requires hundreds or millions of dynamically controlled monochromatic wave sources, spectral control achieves equivalent motion along a single axis using just two continuously driven transducers.

\section{THE WAVE-DRIVEN OSCILLATOR}

Sawtooth oscillation is just one of many modes of motion for this deceptively simple dynamical system. The range of possibilities is illustrated by an idealized model for the dynamics of an object in the acoustic force field. Working in Cartesian coordinates with $\hat{z}$ aligned vertically and $\hat{x}$ directed along the traveling wave, the trapped sphere's horizontal displacement from its equilibrium position, $x(t)$, may be modeled with the equation of motion for a linear harmonic oscillator driven by a harmonic wave:

$$
\frac{d^{2} x}{d t^{2}}+\gamma \frac{d x}{d t}+\omega_{0}^{2} x=\frac{F_{0}}{m} e^{i(k x-\Delta \omega t)},
$$

where $\gamma$ is the sphere's damping rate in air. The harmonicwave model for the driving force is derived from the theory of acoustic forces in Appendix A. The driving force's wave number, $k$, corresponds to the wave number of the traveling wave. The scale of the transverse driving force, $F_{0}$, is proportional to the amplitudes of the driving and trapping waves, and also depends on the sphere's axial position in the acoustic levitator. Measuring typical beads' free oscillations in the trap yields values of the natural frequency ranging from $f_{0}=\omega_{0} /(2 \pi)=3-10 \mathrm{~Hz}$ and damping coefficients in the range $\gamma=2-5 \mathrm{~s}^{-1}$.

The wave-driven harmonic oscillator described by Eq. (1) differs qualitatively from the nonlinear oscillator model that has been used to describe large-amplitude excursions in an acoustic levitator [21]. The most important difference is that the wave-driven oscillator is inherently linear. Its interesting dynamical behavior emerges from the position dependence of the harmonic driving force, which may be viewed as a form of state-dependent driving. Despite the apparent simplicity of Eq. (1) and its evident relevance to experimentally realizable dynamical systems, the wave-driven oscillator appears not to have been discussed previously.

\section{A. Numerical solution}

To explore the phenomenology of the wave-driven oscillator, we recast Eq. (1) in terms of the dimensionless displacement, $\xi(\tau)=k x$, and the dimensionless time, $\tau=$ $\omega_{0} t$, to obtain the nondimensional equation of motion

$$
\xi^{\prime \prime}+b \xi^{\prime}+\xi=\epsilon \exp (i \xi-i \Omega \tau),
$$

where $b=\gamma / \omega_{0}, \Omega=\Delta \omega / \omega_{0}, \epsilon=k F_{0} /\left(m \omega_{0}^{2}\right)$, and primes denote derivatives with respect to $\tau$.

Unlike the canonical driven harmonic oscillator, Eq. (2) does not have analytic solutions. We therefore solve Eq. (2) numerically using the LSODA integrator with 128 steps per driving period, running the integration for 2048 periods to permit transients to decay before recording trajectory data for analysis.

Figure 2 provides a sense of the variety of operating states of the experimental oscillator and the ability of Eq. (2) to capture its behavior. The left column shows experimentally measured phase-space trajectories plotted in nondimensionalized coordinates, $\xi^{\prime}(\xi)$, for three different values of the detuning: (a) $\Delta f=0.1 \mathrm{~Hz}$, which also appears in Fig. 1(c); (b) $\Delta f=1 \mathrm{~Hz}$; and (c) $\Delta f=2.5 \mathrm{~Hz}$. The right column shows corresponding phase-space trajectories that are computed 

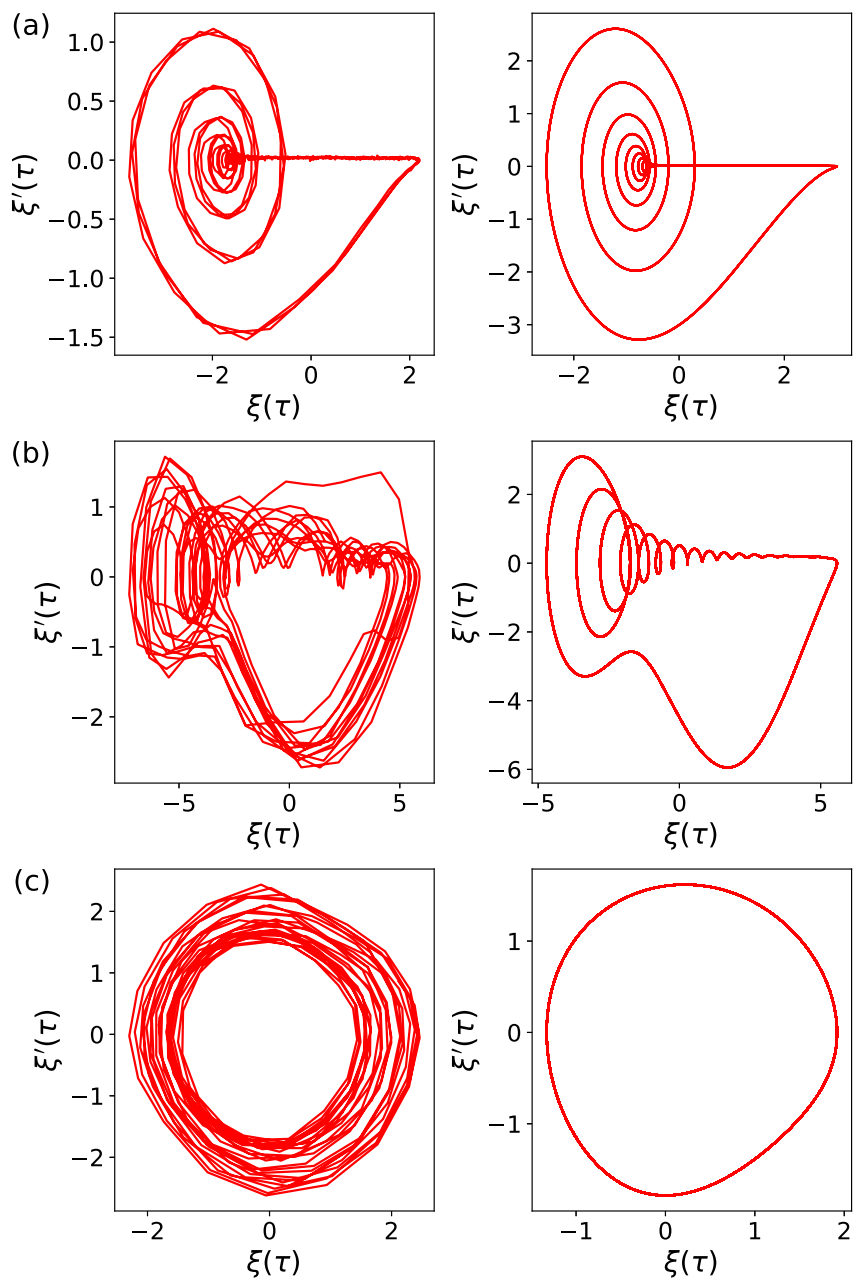

FIG. 2. Comparison between experimentally measured trajectories, $x(t)$, in the left column and numerical solutions to Eq. (2), $\xi(\tau)$, in the right column. Data are plotted as phase-space trajectories, $\xi^{\prime}(\xi)$. (a) $\Delta f=0.1 \mathrm{~Hz}$; data replotted from Fig. $1(\mathrm{c}) . \Omega=0.02$, $\epsilon=3$. (b) $\Delta f=1.0 \mathrm{~Hz} ; \Omega=0.25, \epsilon=5.5$. (c) $\Delta f=2.5 \mathrm{~Hz}$; $\Omega=1, \epsilon=0.7$. All calculations assume $b=0.3$.

with Eq. (2). These are not fits to the experimental data, but rather are comparisons at roughly equivalent ratios of driving frequencies, with fixed damping rate, $b=0.3$, and with driving amplitude adjusted to yield good qualitative agreement.

The agreement between experimental and computed trajectories in Fig. 2 supports the assumptions that link Eq. (1) to the experimental system, including linearity of the waves' superposition. Near-field and edge effects also appear not to be significant, despite the sphere's proximity to the levitating transducer.

Figure 3 presents numerical solutions to Eq. (2) in three dynamical regimes: Fig. 3(a) presents weak driving, $\epsilon=0.1$, in which the particle undergoes nearly harmonic oscillations; Fig. 3(b) presents critical driving, $\epsilon=1$, in which harmonic oscillation gives way to chaos; and Fig. 3(c) presents strong driving, $\epsilon=10$, which is characterized predominantly by sawtooth oscillations. In each case, the dimensionless damping rate is set to $b=\epsilon / 10$.
Panels (d)-(f) of Fig. 3 offer complementary insights into the nature of the wave-driven oscillator's dynamics by presenting the spectral amplitude,

$$
\left|\tilde{x}\left(\omega_{p}\right)\right|=\left|\int_{-\infty}^{\infty} W(t) x(t) e^{i \omega_{p} t} d t\right|,
$$

as a function of driving frequency, $\Delta \omega$, for each of the three sets of driving amplitudes and damping rates from panels (a), (b), and (c), respectively. Each spectrum is computed from 128 driving cycles obtained after transients have decayed. A normalized Blackman-Harris window function, $W(t)$, is used to suppress numerical artifacts due to finite signal duration. The frequency, $\omega_{p}$, being the dual to the time, peaks in $\left|\tilde{x}\left(\omega_{p}\right)\right|$ reflect the frequency content of the harmonic oscillator's response to being driven by a wave at frequency $\Delta \omega$.

The unit-slope diagonal peaks in all three spectrograms in Figs. 3(d)-3(f) represent the system's strong response at the driving frequency, which is the expected behavior of a linear oscillator subjected to harmonic driving. The wave-driven harmonic oscillator also displays features above the diagonal that represent harmonics of the driving frequency as well as features below the diagonal that represent subharmonics. These additional features are not inherent properties of the oscillator, which is linear, but instead arise from the position dependence of wave-mediated driving.

The weakly wave-driven oscillator in Fig. 3(d) displays a spectrum that is composed of (continuous) harmonics of the driving frequency superposed with (discrete) harmonics of the natural frequency, the latter appearing when the driving frequency coincides with a multiple of $\omega_{0}$. These features are captured by a perturbative treatment of Eq (2) in Appendix B.

The spectrum becomes far more complex at critical driving in Fig. 3(e), with harmonics of the natural frequency being joined by subharmonics of the natural frequency and domains of broadband noise.

The remarkable spectrogram in Fig. 3(f) suggests that strong driving supports a sequence of abrupt transitions between dynamical states characterized by subharmonics of the driving frequency. Each state is composed of sawtooth waves whose appearance under strong driving conditions is explained by an idealized model in Appendix C. Whereas such subharmonics are tied to resonancelike harmonics of the fundamental frequency under weak driving conditions in Fig. 3(d), they appear as continuous bands centered around period-n subharmonics of the fundamental under strong driving conditions. The transitions between these dynamical states are not simply aligned with multiples of the fundamental frequency, nor, indeed, are they simply steplike.

\section{B. Period doubling and Fibonacci states}

Figure 4 presents a higher resolution map of the transition between period- 6 and period-7 states that appears as a simple step near $\Omega=2.75$ in Fig. 3(f). The continuous peak near the top of this plot corresponds to the fundamental response, $\omega_{p}=\Delta \omega$. Numbered peaks identify subharmonics within the family of $n=6$ subharmonics on the low-frequency side and $n=7$ subharmonics on the high-frequency side.

Following the states up from low frequency reveals that the $n=6$ state transitions to $n=12$ and $n=24$, following 

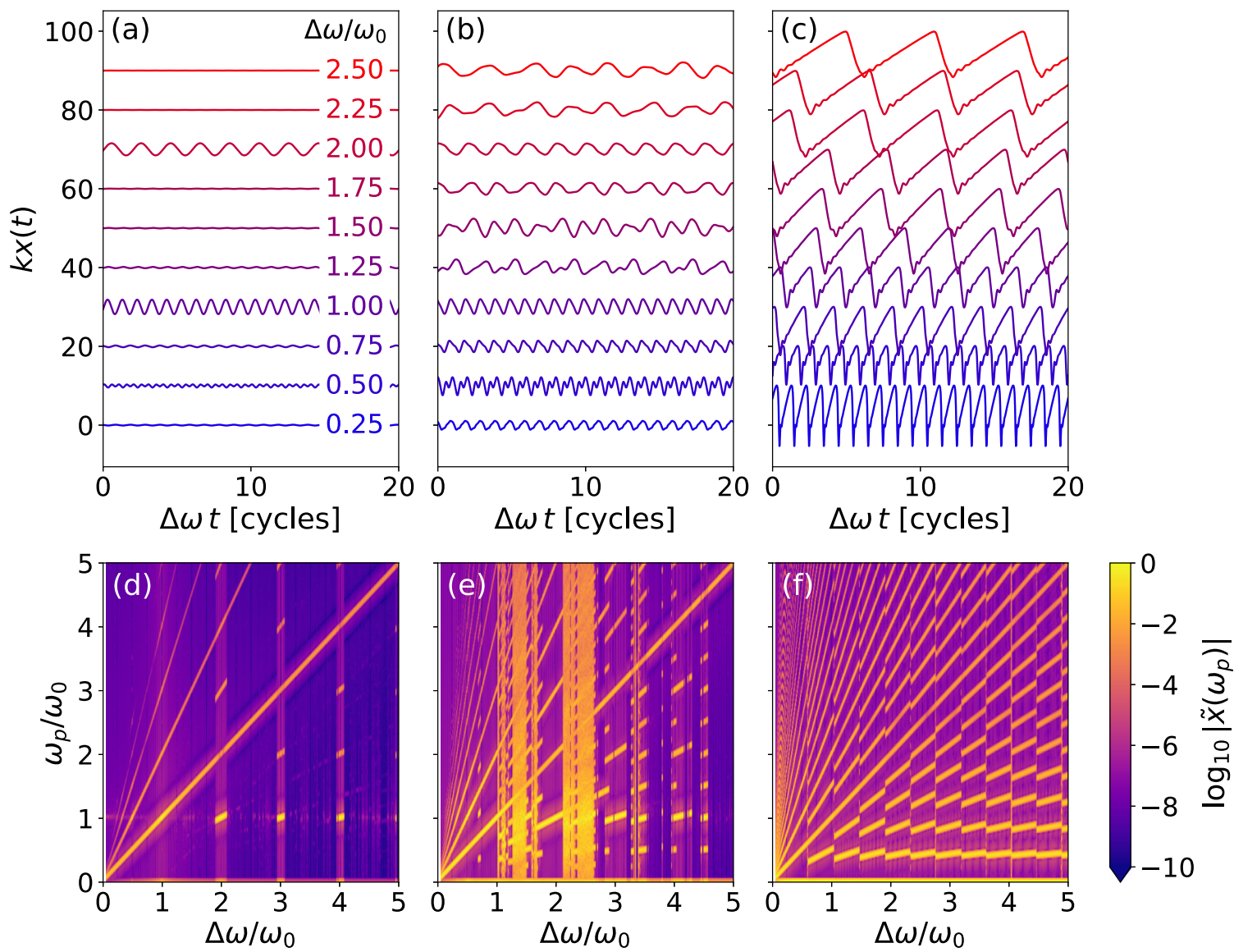

FIG. 3. Computed trajectories for (a) weak driving $(\epsilon=0.1, b=0.01)$, (b) critical driving $(\epsilon=1, b=0.1)$, and (c) strong driving $(\epsilon=10$, $b=1$ ) for driving frequencies ranging from $\Delta \omega=0.25 \omega_{0}$ to $\Delta \omega=2.5 \omega_{0}$. Curves are offset in steps of 10 for clarity and are colored by values of $\Delta \omega / \omega_{0}$. The corresponding power spectra, $\left|\tilde{x}\left(\omega_{p}\right)\right|$, in (d), (e), and (f) reveal markedly different behavior in the different dynamical regimes. These spectrograms are computed at frequency intervals of $0.05 \omega_{0}$ and cover the range from $\Delta \omega=0.1 \omega_{0}$ to $5 \omega_{0}$. Each is normalized to its maximum value.

a period-doubling route to a chaotic state characterized by a broadband power spectrum. Intriguingly, the period-doubling cascade from $n=7$ toward lower frequencies appears to be cut off by the incommensurability between $n=6$ and $n=7$ states.

The phase-space trajectories in Fig. 4 show that the $n=$ 7 state differs from the $n=6$ state by the appearance of an additional epicycle in each cycle. Vertical (white) dotted lines indicate the frequencies at which these trajectories were computed. Rather than appearing continuously, the additional loop in the $n=7$ phase-space trajectory is introduced through a cascade of intermediate states. The intermediate $n=13$ state, for example, is composed of a strict alternation between $n=6$ and $n=7$ states. We therefore recognize $n=13$ as a Fibonacci state composed of the sum of the two states to either side in the frequency representation. Higher-order Fibonacci states also appear in the transition from $n=6$ to $n=7$, involving more complicated sequences. Similar combinations of period-doubling cascades to chaos and Fibonacci cascades appear at the transitions between each of the principal subharmonic states in Fig. 3.

The frequency ranges for the subharmonic states of the strongly wave-driven oscillator remain to be explained, as well as the extraordinarily rich structure of the transitions among these states. The present survey has highlighted just a few of the dynamical states that the wave-driven oscillator can display. Explaining how such a deceptively simple equation of motion can give rise to such varied behavior is presented as an outstanding challenge. In the context of acoustic manipulation, these preliminary investigations highlight the opportunities for achieving sophisticated control over acoustically levitated objects through control over the spectral content of the sound waves instead of dynamic control of their spatial structure.

\section{CONCLUSIONS}

This study introduces a one-dimensional acoustic scanner created from two continuous waves. Adding a third transducer would create opportunities for two-dimensional scanning. More generally, three detuned traveling waves should create a dynamic volumetric force field capable of translating one or more objects through three-dimensional trajectories. Dynamic control over those frequencies promises still more dextrous control in three dimensions while using fewer transducers than a holographic array. Faster motion in this case is achieved with larger detuning, which is technically simpler than rapidly reconfiguring acoustic holograms. 


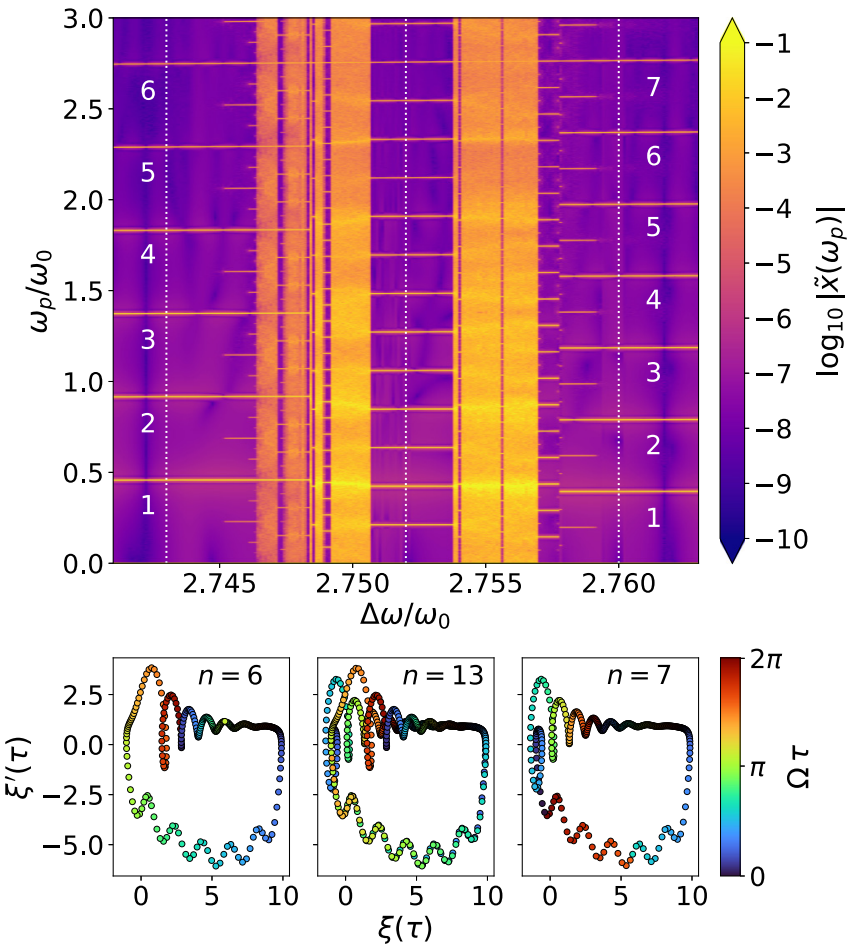

FIG. 4. Detailed view of the transition from the $n=6$ subharmonic state to the $n=7$ state, showing a period-doubling path to chaos and a Fibonacci cascade. Phase-space plots show one cycle of $n=6$ and $n=7$ states bounding the Fibonacci $n=13$ state which appears as a strictly alternating sequence of $n=6$ and $n=7$ trajectories. Trajectory points are colored by periods of the driving frequency as indicated in the color bar. Vertical dotted lines on the spectrogram indicate driving frequencies for the phase-space trajectories.

Realizing the potential of spectral control for practical large-scale manipulation requires a deeper understanding of the dynamics of wave-driven harmonic oscillators. Analytic solutions to Eq. (2), for the minimal example presented in this study, for example, would guide development of practical acoustomechanical scanners. Detuned coaxial acoustic Bessel beams, for example, should act as high-speed tractor beams for transporting material [22] through their analogy to optical conveyors $[23,24]$. Spectral control of acoustic force landscapes [25] also will create new opportunities for influencing the organization of multiparticle systems [26] by introducing a new mechanism for controlling interparticle interactions mediated by the secondary Bjerknes force.

\section{ACKNOWLEDGMENTS}

This work was supported by the MRSEC program of the National Science Foundation under Award No. DMR1420073.

\section{APPENDIX A: THE BEATING ACOUSTIC TRAP AS A WAVE-DRIVEN OSCILLATOR}

The time-averaged acoustic radiation force on a small sphere of radius $a_{p}$ at position $\mathbf{r}$ in a harmonic pressure field $p(\mathbf{r}, t)$ is well approximated by the Gor'kov potential [27,28]:

$$
U(\mathbf{r})=\pi a_{p}^{3} \rho_{m}\left[b_{0} \frac{\left\langle|p(\mathbf{r}, t)|^{2}\right\rangle}{3 \rho_{m}^{2} c_{m}^{2}}-b_{1} \frac{\left\langle|\mathbf{v}(\mathbf{r}, t)|^{2}\right\rangle}{2}\right] .
$$

The sphere's coupling to the sound wave depends on its compressibility relative to the medium through

$$
b_{0}=1-\frac{\rho_{m} c_{m}^{2}}{\rho_{p} c_{p}^{2}}
$$

and its relative density through

$$
b_{1}=\frac{2\left(\rho_{p}-\rho_{m}\right)}{2 \rho_{p}+\rho_{m}} .
$$

The symbols $\rho_{j}$ and $c_{j}$ denote density and sound speed, respectively, in the material denoted by $j$. Subscripts $p$ and $m$ refer to the particle and surrounding medium, respectively. In the system under study, the acoustic contrast between air and the styrofoam sphere is large enough that $b_{0} \approx b_{1} \approx 1$. Angle brackets in Eq. (A1) signify time averages.

The velocity field is related to the pressure field in linear sound waves by Newton's second law,

$$
\rho_{m} \frac{\partial \mathbf{v}}{\partial t}+\nabla p=0 .
$$

For a harmonic wave at frequency $\omega$, therefore, the pressure acts as a scalar potential for the velocity,

$$
\mathbf{v}(\mathbf{r}, t)=-\frac{i}{\omega \rho} \nabla p .
$$

Equations (A1) and (A4) define the acoustic force field established by a specified pressure field, $p(\mathbf{r}, t)$.

The wave-driven oscillator is subject to two harmonic pressure fields: a standing wave,

$$
p_{1}(\mathbf{r}, t)=u_{1} \sin \left(k_{1} z\right) e^{-i \omega_{1} t},
$$

at frequency $\omega_{1}$ that forms an acoustic trap and a traveling wave,

$$
p_{2}(\mathbf{r}, t)=u_{2} e^{i k_{2} x} e^{-i \omega_{2} t},
$$

at frequency $\omega_{2}$ that provides the driving.

To ensure that the amplitudes $u_{1}$ and $u_{2}$ are real valued we absorb spatial and temporal phase differences into the definitions of $x$ and $t$.

The total pressure field, $p(\mathbf{r}, t)=p_{1}(\mathbf{r}, t)+p_{2}(\mathbf{r}, t)$, has an intensity

$$
|p(\mathbf{r}, t)|^{2}=\left|p_{1}\right|^{2}+\left|p_{2}\right|^{2}+2 \operatorname{Re}\left\{p_{1}^{*} p_{2}\right\}
$$

that includes the time-independent intensities of the component waves,

$$
\left|p_{1}\right|^{2}+\left|p_{2}\right|^{2}=u_{1}^{2} \sin ^{2}\left(k_{1} z\right)+u_{2}^{2},
$$

and a time-dependent cross term,

$$
\begin{aligned}
2 \operatorname{Re}\left\{p_{1}^{*} p_{2}\right\}= & 2 u_{1} u_{2} \operatorname{sinc}(\phi) \\
& \times \sin \left(k_{1} z\right) \cos \left(k_{2} x-\Delta \omega t-\phi\right),
\end{aligned}
$$

that oscillates at the difference frequency, $\Delta \omega=\omega_{2}-\omega_{1}$. The phase offset, $\phi=\pi \Delta \omega / \omega$, depends on the detuning of the two waves relative to the center frequency $\omega=\left(\omega_{1}+\right.$ $\left.\omega_{2}\right) / 2$ and contributes to the amplitude of the cross term. 
Choosing the complex-exponential form for the time dependence of $p_{1}(\mathbf{r}, t)$ and $p_{2}(\mathbf{r}, t)$ omits a sum-frequency component that might otherwise appear in the superposition, $p(\mathbf{r}, t)$. This choice does not affect the acoustic force field because a high-frequency contribution to $|p(\mathbf{r}, t)|^{2}$ oscillating at $2 \omega+\Delta \omega$ would be suppressed by the time average in Eq. (A1).

Pursuing a similar line of reasoning for the velocity yields

$$
\begin{gathered}
|\mathbf{v}(\mathbf{r}, t)|^{2}=\left|\mathbf{v}_{1}\right|^{2}+\left|\mathbf{v}_{2}\right|^{2}+2 \operatorname{Re}\left\{\mathbf{v}_{1}^{*} \cdot \mathbf{v}_{2}\right\} \\
=\frac{u_{1}^{2}}{\rho_{m}^{2} c_{m}^{2}} \cos ^{2}\left(k_{1} z\right)+\frac{u_{2}^{2}}{\rho_{m}^{2} c_{m}^{2}}
\end{gathered}
$$

with the cross term vanishing because the two waves are orthogonal. Dropping constant terms, we obtain a timedependent potential,

$$
\begin{aligned}
U(\mathbf{r}, t)= & A \cos ^{2}\left(k_{1} z\right) \\
& +B \sin \left(k_{1} z\right) \cos \left(k_{2} x-\Delta \omega t\right),
\end{aligned}
$$

that includes an axial trapping potential whose energy scale,

$$
A=\frac{1}{6} u_{1}^{2} \frac{\pi a_{p}^{3}}{\rho_{m} c_{m}^{2}},
$$

is set by the intensity of the standing wave, and a timedependent contribution that oscillates at the beat frequency because it arises from the interference between the standing wave and the traveling wave. The energy scale for this time-dependent interaction depends on the amplitudes of both waves:

$$
B=\frac{2}{3} u_{1} u_{2} \frac{\pi a_{p}^{3}}{\rho_{m} c_{m}^{2}} .
$$

In the absence of external forces, the trapping potential due to the standing wave would tend to localize the particle near $k_{1} z=\pi / 2$, which would effectively suppress the transverse driving force. Gravity, however, displaces the particle downward to

$$
z_{0} \approx-\frac{1}{2 k_{1}} \arcsin \left(\frac{8 \rho_{p} \rho_{m} c^{2} g}{5 k_{1} u_{1}^{2}}\right),
$$

where $g$ is the acceleration due to gravity. The resulting transverse force then becomes

$$
F(\mathbf{r}, t)=F_{0} \sin \left(k_{2} x-\Delta \omega t\right),
$$

with the force scale $F_{0}=-k_{2} B \sin \left(k_{1} z_{0}\right)$. Equation (A13) motivates the choice of a wavelike driving force for the right-hand side of Eq. (1).

In the interest of clarity, this analysis has not accounted for the transverse trapping force arising from transverse gradients in the vertical standing wave, but rather has treated the standing wave as planelike near the trapping plane. A more complete treatment yields an expression comparable to Eq. (A13) with corrections to the scale factor, $F_{0}$. Interestingly, wavelike driving in this system appears to rely on gravity to displace the trapped particle. We predict therefore that the effects observed in this study would not occur under density-matched conditions or in microgravity.

This analysis also excludes the scattering force that the transverse traveling wave exerts, which is not accounted for by the Gor'kov potential [14,25,29]. This force arises from higher-order terms in the acoustic multipole expansion and so is much weaker than the other forces acting on the wavedriven oscillator. Its principal effect is to apply a nearly constant and uniform force on the levitated sphere that slightly shifts its equilibrium position in the trap.

\section{APPENDIX B: WEAK DRIVING IN THE UNDERDAMPED REGIME}

The wave-driven oscillator does not behave like a standard periodically driven harmonic oscillator even when the driving is weak. This is evident in the appearance of harmonics, resonancelike subharmonics, and their superpositions in Fig. 3(d). We explain this behavior by treating Eq. (2) perturbatively in the limit of weak driving $(\epsilon<1)$ in the inertial regime where damping may be ignored $(b \ll 1)$. Under these conditions, Eq. (2) reduces to

$$
\xi^{\prime \prime}(\tau)+\xi(\tau) \approx \epsilon e^{i \xi(\tau)-i \Omega \tau} .
$$

Substituting a trial solution

$$
\xi(\tau)=\xi_{0}(\tau)+\epsilon \xi_{1}(\tau)+\epsilon^{2} \xi_{2}(\tau)+\ldots,
$$

with initial conditions $\xi_{n}(0)=\xi_{n}^{\prime}(0)=0$ yields for the first few terms

$$
\begin{aligned}
\xi_{0}(\tau)= & 0, \\
\xi_{1}(\tau)= & \frac{e^{-i \Omega \tau}}{1-\Omega^{2}}+\frac{1}{2}\left(\frac{e^{-i \tau}}{\Omega-1}-\frac{e^{i \tau}}{\Omega+1}\right), \\
\xi_{2}(\tau)= & i \frac{e^{-2 i \Omega \tau}}{\left(\Omega^{2}-1\right)\left(4 \Omega^{2}-1\right)} \\
& -\frac{i}{2 \Omega}\left[\frac{e^{-i \tau}}{(\Omega-2)(2 \Omega-1)}-\frac{e^{i \tau}}{(\Omega+2)(2 \Omega+1)}\right] \\
& -\frac{i}{2 \Omega}\left[\frac{e^{-i(\Omega+1) \tau}}{(\Omega-1)(\Omega+2)}-\frac{e^{-i(\Omega-1) \tau}}{(\Omega+1)(\Omega-2)}\right] .
\end{aligned}
$$

The first-order contribution in Eq. (B3b) includes a response at the the driving frequency, $\Omega$, which is the expected behavior for a driven harmonic oscillator. It also includes a response at the natural frequency, $\omega_{p}=\omega_{0}$, that appears regardless of the driving frequency, and is resonantly enhanced when $\Omega= \pm 1$. This feature is not surprising because transients at the natural frequency do not decay when $b=0$. The response at $\omega_{p}=\omega_{0}$ appears in Fig. 3(d) as a horizontal streak with a resonant peak on the main diagonal when $\Delta \omega=\omega_{0}$.

The second-order contribution from Eq. (B3c) is more interesting. It includes the second harmonic of the driving frequency, which appears in Fig. 3(d) as a continuous diagonal streak at $\omega_{p}=2 \Delta \omega$. The second term in Eq. (B3c) contributes to the oscillator's response at the natural frequency, with additional resonant enhancement at $\Omega= \pm 2$ and $\Omega= \pm 1 / 2$. The third term describes sum- and difference-frequency responses at $\omega_{p}=\Delta \omega \pm \omega_{0}$ that are resonantly enhanced near $\Omega=2$. This contribution accounts for the frequency dependence of the observed peaks in Fig. 3(d) near $\omega_{p}=\omega_{0}$ and $\omega_{p}=3 \omega_{0}$ for driving frequencies in the vicinity of $\Delta \omega=2 \omega_{0}$.

Higher-order terms introduce corresponding harmonics of the driving frequency as well as the sum- and 
difference-frequency responses that account for the grid of discrete resonant peaks in Fig. 3(d).

\section{APPENDIX C: STRONG DRIVING IN THE OVERDAMPED REGIME}

The sawtooth oscillations presented in Fig. 3(c) arise under strong driving conditions, $\epsilon>1$, and feature long stretches of nearly linear motion punctuated by abrupt changes in direction. The equation of motion from Eq. (2) may be approximated in these weakly inertial passages by the transcendental equation

$$
\xi=\epsilon \sin (\xi-\Omega \tau) .
$$

For large $\epsilon$, the object follows the driving wave until $\xi \approx \epsilon$, at which point the restoring force overcomes the driving force and the object snaps back. This occurs at $\tau=\epsilon / \Omega$ which suggests the fundamental oscillation frequency

$$
\omega_{p}=2 \pi \Delta \omega \frac{m \omega_{0}^{2}}{k F_{0}}
$$

can be substantially lower than the driving frequency $\Delta \omega$ because the driving wave may have to advance by several wavelengths before the object can break free.

Once the object is free, it travels from $\xi=\epsilon$ back toward $\xi=0$ until it once again encounters a region of the traveling wave that can stop it and reverse its motion. Equation $(\mathrm{C} 1)$ does not account for the acceleration that occurs during the oscillator's recovery. It therefore does not accurately predict transition frequencies between the oscillator's operating points, nor does it describe the complicated hierarchy of dynamical states near transitions.
[1] S. Inoue, S. Mogami, T. Ichiyama, A. Noda, Y. Makino, and H. Shinoda, Acoustical boundary hologram for macroscopic rigidbody levitation, J. Acoust. Soc. Am. 145, 328 (2019).

[2] A. Marzo and B. W. Drinkwater, Holographic acoustic tweezers, Proc. Natl. Acad. Sci. USA 116, 84 (2019).

[3] K. Melde, A. G. Mark, T. Qiu, and P. Fischer, Holograms for acoustics, Nature (London) 537, 518 (2016).

[4] T. Kozuka, K. Yasui, T. Tuziuti, A. Towata, and Y. Iida, Noncontact acoustic manipulation in air, Jpn. J. Appl. Phys. 46, 4948 (2007).

[5] J. Wu, Acoustical tweezers, J. Acoust. Soc. Am. 89, 2140 (1991).

[6] D. Baresch, J.-L. Thomas, and R. Marchiano, Observation of a Single-Beam Gradient Force Acoustical Trap for Elastic Particles: Acoustical Tweezers, Phys. Rev. Lett. 116, 024301 (2016).

[7] G. Thalhammer, R. Steiger, M. Meinschad, M. Hill, S. Bernet, and M. Ritsch-Marte, Combined acoustic and optical trapping, Biomed. Opt. Express 2, 2859 (2011).

[8] D. G. Grier, A revolution in optical manipulation, Nature (London) 424, 810 (2003).

[9] J. Leach, G. Sinclair, P. Jordan, J. Courtial, M. J. Padgett, J. Cooper, and Z. J. Laczik, 3D manipulation of particles into crystal structures using holographic optical tweezers, Opt. Express 12, 220 (2004).

[10] A. Marzo, S. A. Seah, B. W. Drinkwater, D. R. Sahoo, B. Long, and S. Subramanian, Holographic acoustic elements for manipulation of levitated objects, Nat. Commun. 6, 8661 (2015).

[11] R. Hirayama, D. M. Plasencia, N. Masuda, and S. Subramanian, A volumetric display for visual, tactile and audio presentation using acoustic trapping, Nature (London) 575, 320 (2019).

[12] T. Fushimi, B. W. Drinkwater, and T. L. Hill, What is the ultimate capability of acoustophoretic volumetric displays? Appl. Phys. Lett. 116, 244101 (2020).

[13] B. W. Drinkwater, Dynamic-field devices for the ultrasonic manipulation of microparticles, Lab Chip 16, 2360 (2016).

[14] P. J. Westervelt, Acoustic radiation pressure, J. Acoust. Soc. Am. 29, 26 (1957).

[15] Nonlinear acoustics in fluids, edited by R. T. Beyer (Van Nostrand Reinhold Company, New York, 1984).

[16] See Supplemental Material at http://link.aps.org/supplemental/ 10.1103/PhysRevResearch.3.013079 for a video of an acoustically levitated particle undergoing wave-driven oscillations.

[17] J. C. Crocker and D. G. Grier, Methods of digital video microscopy for colloidal studies, J. Colloid Interface Sci. 179, 298 (1996).

[18] D. Allan, C. van der Wel, N. Keim, T. A. Caswell, D. Wieker, R. Verweij, C. Reid, Thierry, L. Grueter, K. Ramos, apiszcz, zoeith, R. W. Perry, F. Boulogne, P. Sinha, pfigliozzi, N. Bruot, L. Uieda, J. Katins, H. Mary, and A. Ahmadia, Trackpy v0.4.2, 2019.

[19] F. Zheng, Y. Li, H.-S. Hsu, C. Liu, C. Tat Chiu, C. Lee, H. Ham Kim, and K. K. Shung, Acoustic trapping with a high frequency linear phased array, Appl. Phys. Lett. 101, 214104 (2012).

[20] Y. Ochiai, T. Hoshi, and J. Rekimoto, Three-dimensional midair acoustic manipulation by ultrasonic phased arrays, PloS One 9, e97590 (2014).

[21] T. Fushimi, T. Hill, A. Marzo, and B. Drinkwater, Nonlinear trapping stiffness of mid-air single-axis acoustic levitators, Appl. Phys. Lett. 113, 034102 (2018).

[22] P. L. Marston, Axial radiation force of a Bessel beam on a sphere and direction reversal of the force, J. Acoust. Soc. Am. 120, 3518 (2006).

[23] T. Čižmár, V. Garcés-Chávez, K. Dholakia, and P. Zemánek, Optical conveyor belt for delivery of submicron objects, Appl. Phys. Lett. 86, 174101 (2005).

[24] D. B. Ruffner and D. G. Grier, Optical Conveyors: A Class of Active Tractor Beams, Phys. Rev. Lett. 109, 163903 (2012).

[25] M. A. Abdelaziz and D. G. Grier, Acoustokinetics: Crafting force landscapes from sound waves, Phys. Rev. Res. 2, 013172 (2020).

[26] M. X. Lim, A. Souslov, V. Vitelli, and H. M. Jaeger, Cluster formation by acoustic forces and active fluctuations in levitated granular matter, Nat. Phys. 15, 460 (2019).

[27] L. P. Gor'kov, On the forces acting on a small particle in an acoustical field in an ideal fluid, Sov. Phys. Dokl. 6, 773 (1962).

[28] H. Bruus, Acoustofluidics 7: The acoustic radiation force on small particles, Lab Chip 12, 1014 (2012).

[29] G. T. Silva, Acoustic radiation force and torque on an absorbing compressible particle in an inviscid fluid, J. Acoust. Soc. Am. 136, 2405 (2014).

Correction: A minor error in Eq. (A12) has been fixed. 